\title{
Tai Chi training can improve postural control
}

\author{
Orlando FERNANDES ${ }^{*}$, \& Fábio BASILIO ${ }^{2}$ \\ ${ }^{1}$ Sports and Health Department, Sciences and Technology School, University of Évora (Portugal) \\ ${ }^{2}$ Zen Concept Clinic, Every Body Health Club (Portugal)
}

\section{Introduction}

A traditional Chinese form of physical activity is Tai Chi Chuan (TCC). The basic TCC exercise is a series of graceful movements linked together in a continuous sequence so that the body is constantly shifting from foot to foot, with a lower center of gravity. Deep breathing and mental concentration are required to achieve harmony between body and mind in TCC exercise. There is evidence that TCC exercise program may improve postural control (Wayne, et al., 2004; Kendrick, et al., 2014) and minimize the risk of falls (Park, et al., 2013). At present, few data exist to support the contention that TCC specifically targets the impairments, functional limitations, disability, and quality of life associated with peripheral vestibulopathy, main reason for balance disorders (Wayne, et al., 2004). The objective of this study was to examine whether 4 months of TCC practice could improve balance control in the healthy subjects. This study explored whether the Tai Chi exercise program would affect the ability to minimize postural sway and changes in complexitybased COP measures.

\section{Methodology}

A group of 10 healthy adults (mean age $\pm s d=60 \pm 4.9$ years) were recruited from two Tai Chi club and as selection criterion the participants had to perform 15 sessions of 1-hour class and without any history of fall or injury during the study. The intervention phase lasted 4 months between January 2016 and April 2016. All subjects were evaluated under four postural conditions for postural stability sequentially as follows: (1) Both feet eyes open (BE0); (2) Both feet eyes closed; (3/4) One leg stand eyes open (OLS -left and right leg). Participants stood in bare feet on the force plate (BERTEC 4060, Columbus, USA) with a comfortable foot placement during 60 seconds. The data were passed through a 4-order zero phase Butterworth low-pass filter with $5-\mathrm{Hz}$ cut-off frequency and down sampled at $100 \mathrm{~Hz}$. From the last 30 seconds of the time series in anteriorposterior and medial-lateral direction we computed the measures of postural steadiness. Total excursion (DOT); the average velocity of the COP (MVELO); the 95\% confidence ellipse area (AREA$\mathrm{CE}$ ) and a nonlinear measure the approximated Entropy of a time series as measured of complexity.

\section{Results}

If we look the traditional measures of postural control of and starting from the hypothesis that the decrease of the sway values of the COP can translate a better ability to control. Our results show that the two groups improve generally their ability to keep quiet stand especially with closed eyes and one leg stand. Mean \pm sd-improvement (\%): BEO/DOT (mm) $=341,607 \pm 139,492 ; 217,610 \pm$ 61,121 (36,3\%); BEO/MVELO (mm/s) = 13,664 \pm 5,580; 8,704 $\pm 2,445 ;(36,3 \%) ;$ OLS left/DOT = $462,436 \pm 121,034 ; 382,483 \pm 88,632(17,3 \%) ;$ OLS right/DOT $=516,335 \pm 245,709 ; 427,474 \pm$ 
$132,185(17,2 \%)$. When we eliminate the sensory input of vision, the participants improve in all parameters revealing a greater capacity of concentration and an increased a sensory motor sensitivity. The group which only held Tai Chi with other physical activity obtained the best results than the participants who only did Tai Chi without other physical activities.

\section{Discussion and conclusion}

Measures from postural control revealed greater stability after Tai Chi training among subjects in all training group but little change in stability among subjects that use only Tai Chi training. Less sway during quiet standing reveals better capacity in postural control maybe Tai Chi may promote more confidence after training (Wolf et al. 1997).The particular characteristics of Tai Chi exercise, which emphasizes control over one's displacement of body mass, postural alignment, and range of motion of joints and muscles of the lower body, may be part of a larger mechanism responsible for specific gains in leg strength and postural stability (Li, et al., 2005). As Kendrick et al. (2014) we can assume that Tai Chi training in older persons can decrease the number of falls and injurious falls by participating in a regular program of Tai Chi. The above findings indicated that 15 sessions of Tai Chi training are sufficient to improve balance control in the elderly subjects and change behaviour complexity that may indicate a change in the control strategies postural. Another major conclusion is that the Tai Chi, in conjunction with other physical activities, enhances the improvement in the postural control.

\section{References}

Kendrick, D., Kumar, A., Carpenter, H., Zijlstra, G. A., Skelton, D. A., Cook, J. R., et al. (2014). Exercise for reducing fear of falling in older people living in the community. The Cochrane database of systematic reviews, 28(11), CD009848.

Li, F., Harmer, P., Fisher, K. J., McAuley, E., Chaumeton, N., Eckstrom, E., et al. (2005). Tai Chi and fall reductions in older adults: a randomized controlled trial. The journals of gerontology. Series A, Biological sciences and medical sciences, 60(2), 187-194.

Park, M., \& Song, R. (2013). [Effects of Tai Chi on fall risk factors: a meta-analysis]. Journal of Korean Academy of Nursing, 43(3), 341-351.

Wayne, P. M., Krebs, D. E., Wolf, S. L., Gill-Body, K. M., Scarborough, D. M., McGibbon, C. A., et al. (2004). Can Tai Chi improve vestibulopathic postural control? Archives of physical medicine and rehabilitation, 85(1), 142-152.

Wolf, S. L., Barnhart, H. X., Ellison, G. L., \& Coogler, C. E. (1997). The effect of Tai Chi Quan and computerized balance training on postural stability in older subjects. Atlanta FICSIT Group. Frailty and Injuries: Cooperative Studies on Intervention Techniques. Physical therapy, 77(4), 371-381; discussion 382-374.

Key words: Tai Chi Chuan; exercise; posture; balance; elderly.

The authors thank Évora Every Body Health Club

Rev. Artes Marciales Asiát., 11(2s), 42-43 2016 